\title{
Exploring the diameter and surface dependent conformational changes in carbon nanotube-protein corona and the related cytotoxicity
}

\author{
Xingchen Zhao ${ }^{\mathrm{a}}$, Dawei Lu ${ }^{\mathrm{a}}$, Fang Hao ${ }^{\mathrm{a}}$, Rutao Liu ${ }^{\mathrm{b}, *}$ \\ a State Key Laboratory of Environmental Chemistry and Ecotoxicology, Research Center for Eco-Environmental Sciences, \\ Chinese Academy of Sciences, Beijing 100085, PR China \\ ${ }^{\mathrm{b}}$ Shandong Key Laboratory of Water Pollution Control and Resource Reuse, School of Environmental Science and Engineering, \\ Shandong University, China-America CRC for Environment \& Health, Jinan 250100, PR China
}

\section{H I G H L I G H T S}

- CNT diameter and surface area govern the stability of adsorbed proteins.

- More BSA was loaded and destabilized on smaller CNTs.

- Protein corona reduces the cytotoxicity of CNTs
G R A P H I C A L A B S T R A C T

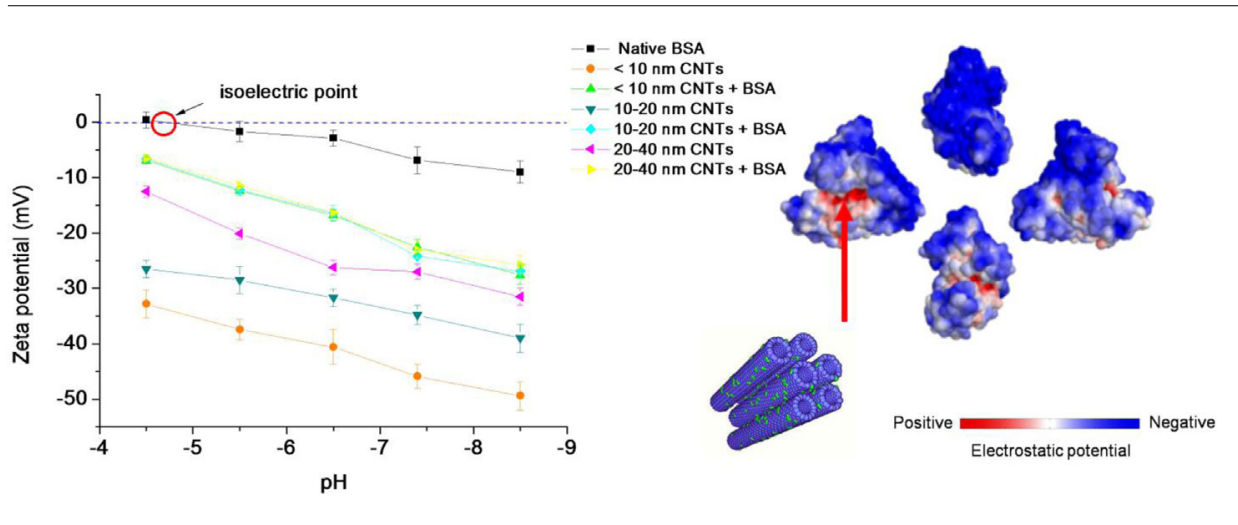

\begin{abstract}
A B S T R A C T
In this work, we investigated and compared carbon nanotubes (CNTs) of different diameters regarding their interaction with bovine serum albumin (BSA) and their ability to alter protein structure. BSA was exposed to CNT solutions, and the effects were assessed by utilizing fluorescence spectroscopy, UV-vis absorption spectroscopy, circular dichroism (CD) spectroscopy, transmission electron microscopy (TEM), bichinchoninic acid (BCA) and zeta-potential measurement assays. We demonstrate that CNT diameter and surface area play key roles in influencing the stability of adsorbed proteins. Results showed that the secondary and tertiary structural stability of BSA decreased upon adsorption onto CNTs, with greater decrease on smaller-diametered nanotubes. Besides, more protein was loaded onto CNTs with small diameter, reducing the cytotoxicity. This study, therefore, provides fundamental information for the influence of CNT diameter and surface on protein behavior, which may be helpful to understand toxic effects of CNTs and prove beneficial for developing novel biomedical devices and safe use of nanomaterials.
\end{abstract}

(c) 2015 Elsevier B.V. All rights reserved.

\footnotetext{
* Corresponding author. Tel.: +86531 88364868; fax: +86 53188364868

E-mail address: rutaoliu@sdu.edu.cn (R. Liu).
}

\section{Introduction}

The discovery of numerous carbon nanomaterials has created a new field of vision to the rapid development of 
nanotechnology especially after the discovery of carbon nanotubes (CNTs) by Iijima [1]. Keen interest due to their unique electrical and mechanical properties is now leading to mass production and commercial application of nanomaterials, for example, nanotechnology, nanoelectronics, and composite materials [2,3]. Researchers showed that bioelectrochemical reactions can be driven by attaching proteins onto the surface of CNTs and that wellcontrolled aligned CNTs can be applied as immobilization matrices and as mediators for the development of biosensor devices [4]. Not just this, researchers also focus on how to form stable colloidal dispersions in various solvents including physiological solutions, in which solubility of CNTs is extremely small. Such soluble CNTs were expected to be promising as the basis for potential biotechnological and medical applications, in particular, utilized for DNA, proteins, and drug delivery [5].

The wide production, use and disposal of CNTs will inevitably lead to their exposure to human beings. Recent years, uncertainty over the potential detrimental effects of CNTs on human health and the environment has caused wide attention, especially the editorial articles on bio-effects, environmental and physical influences of nanoscaled particles was published on Science [6] and Nature [7].

Proteins are chief actors and fundamental components of all living cells. By studying the structures of proteins, we are better able to understand how they function normally and how some proteins with abnormal shapes can cause disease. Thus to investigate the interaction of protein and CNTs would be of great value and be potential to reveal the mechanism of CNT toxicity. However, data for CNT toxicity at a protein level are still scarce so far. Zhang et al. found that functionalized CNTs specifically bind to $\alpha-$ chymotrypsin's catalytic site and regulate its enzymatic function [8]. Mu presented the conjugation of four proteins onto diverse multi-walled carbon nanotubes (MWCNTs). The three-dimentional structure of BSA changed followed by exposure of hydrophobic groups [9]. In our previous study we showed that MWCNTs could interact with BSA through mainly electrostatic forces and could cause secondary and tertiary structure alteration of the protein [10].

Here, we used MWCNTs with diverse diameters which were shortened, and functionalized by strong-oxidative solution. We emphasized the diameter-dependent effect of CNTs with BSA, which is the major component of blood plasma and serves as a carrier of chemical substances. Fluorescence spectroscopy was used to detect the microenvironment alteration of BSA's fluorescent residues caused by CNTs. and ultraviolet-visible absorption spectroscopy was utilized to analyze protein's conformation and backbone changes. Furthermore, secondary and tertiary structural transformations were monitored by CD spectroscopy. We demonstrate quantitatively that the heart-shaped protein undergoes different degrees of unfolding as a function of CNT diameter. This work provides new insights into the impact of CNT diameter and surface area upon the secondary and tertiary structural stability of adsorbed protein as well as concerning cytotoxicity.

\section{Experimental}

\subsection{Reagents and apparatus}

MWCNTs (<10 nm, 10-20 nm and 20-40 nm) were bought from Shenzhen Nanotech Port Co., Ltd. (Shenzhen, China). All MWCNTs were synthesized by chemical vapor deposition. The purity was higher than $90 \%$, and the catalyst residue was less than $0.2 \%$. $\mathrm{NaH}_{2} \mathrm{PO}_{4}, \mathrm{Na}_{2} \mathrm{HPO}_{4}, \mathrm{HNO}_{3}, \mathrm{H}_{2} \mathrm{SO}_{4}$ and $\mathrm{NaOH}$ were all purchased from Tianjin Tianda Chemical Reagent Co., Ltd. (Tianjin, China). BSA was bought from Sigma Chemical Co. (St. Louis, M.O.) without further purification, and was dissolved in ultrapure water to form a $1.0 \times 10^{-5} \mathrm{~mol} / \mathrm{L}$ solution, then preserved at $0-4{ }^{\circ} \mathrm{C}$ for later use.
All solutions were prepared with ultrapure water (18.25 M $\Omega$ ) and adjusted the $\mathrm{pH}$ to 7.4 using $0.2 \mathrm{M}$ phosphate buffered saline (PBS).

In this work, $8400 \mathrm{~S}$ Fourier transform infrared (FTIR) spectroscopy (Shimadzu, Japan) and Q600SDT thermogravimetric analyzer (TA Instruments, U.S.) were used for characterization of CNTs. The ultraviolet/visible absorbance spectra (UV-vis) were recorded on a Shimadzu UV-2450 spectrometer (Shimadzu, Japan) in a $1 \mathrm{~cm}$ quartz cell with reference to proper solutions. Transmission electron microscopy (TEM) images were captured using a JEM-2100 transmission electron microscope (JEOL Inc., Japan). Size of MWCNTs was characterized by a BI-200SM Dynamic/static Laser scattering system (Brooken Haven, USA.). Steady-state fluorescence spectra were taken using an FL-4600 fluorescence spectrophotometer (Hitachi Co., Ltd., Japan). The emission spectra were monitored in the range of $290-410 \mathrm{~nm}$ with the fixed slit width of $5 \mathrm{~nm}$. Inner filter effect which is caused by the absorption of both excitation and emission radiation of the sample was taken into account in this system. CD spectra were measured by a J-810 Spectropolarimeter (Jasco, Japan) under constant nitrogen flush. The far-UV region was scanned between 200 and $240 \mathrm{~nm}$ with an average of two scans and also a bandwidth of $5 \mathrm{~nm}$. BET surface area measurements were performed with a Quadrasorb SI unit (Quantachrome, USA) using nitrogen adsorption at $353 \mathrm{~K}$. PBS buffer were used to adjust the $\mathrm{pH}$ to the desired value. The $\zeta$ potential was measured using a Malvern ZetaSizer NanoSeries (Malvern Instruments, $\mathrm{UK})$ at $298 \mathrm{~K}$.

\subsection{Preparation of soluble CNTs with diverse diameters}

To remove the impurities, a pristine sample of nanotubes $(0.8 \mathrm{~g})$ was first immersed in $1.2 \mathrm{~L}$ of $2 \mathrm{M}$ nitric acid and stirred for $24 \mathrm{~h}$. The solids were then washed twice with $2 \mathrm{~L}$ ultrapure water. The purified tubes were then being suspended in $200 \mathrm{~mL}$ of a $3: 1$ mixture of concentrated $\mathrm{H}_{2} \mathrm{SO}_{4} / \mathrm{HNO}_{3}$ (v:v) and stirred at reflux $\left(60-70{ }^{\circ} \mathrm{C}\right)$ for $24 \mathrm{~h}$. The resultant suspension was poured in $800 \mathrm{~mL}$ of water afterwards. After being filtered and washed with $10 \mathrm{mM} \mathrm{NaOH}$ solution on a 220-nm pore filter membrane, larger MWCNTs were dried in vacuum to obtain solvable powder. We suspended the as-prepared MWCNTs at a density of $0.15 \mathrm{mg} / \mathrm{mL}$ in water then diluted into different concentrations followed by absorbance measurements at $907 \mathrm{~nm}$. Absorbance vs concentration working curve was plotted as demonstrated in our previous work [10]. MWCNT solution of a certain concentration was centrifuged and the supernatant was obtained. The concentration of the surfactant with well dispersed MWCNTs was determined according to the absorbance of the aqueous solution.

\subsection{Protein adsorption onto CNTs}

For protein adsorption experiments, CNT stock solutions $(0.1 \mathrm{~g} / \mathrm{L})$ were shaken and subjected to sonication for $5 \mathrm{~min}$. CNTs were then exposed to $0.065 \mathrm{~g} / \mathrm{LBSA}$ solution with $20 \mathrm{mM}$ phosphate buffer to reach a final concentration of $10 \mathrm{mg} / \mathrm{L}$ in $10 \mathrm{~mL}$ Corning centrifuge tubes. The mixtures were then shaken on a platform shaker for $1 \mathrm{~h}$ at $150 \mathrm{rpm}$ and $25^{\circ} \mathrm{C}$. After incubation, the mixtures were centrifuged at $7000 \mathrm{rpm}$ and the supernatant was removed for protein concentration detection. BCA reagent box was bought from Biocolor BioScience \& Technology Company (Shanghai, China) and was used for determination of protein solution concentration.

\subsection{Cell culture and cell viability test by MTT assay}

Primary astrocytes line was obtained by the method from Frangakis [11]. The cells were maintained in DMEMF12 medium (Hyclone, USA) supplemented with fetal bovine serum (10\%, 
A

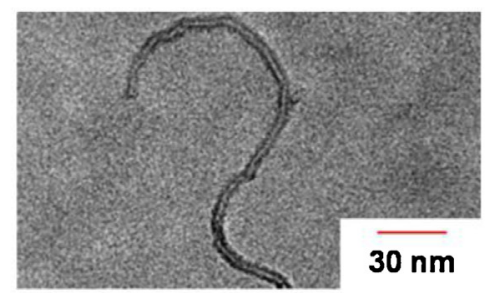

B

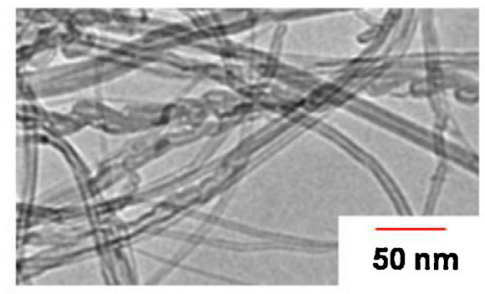

C

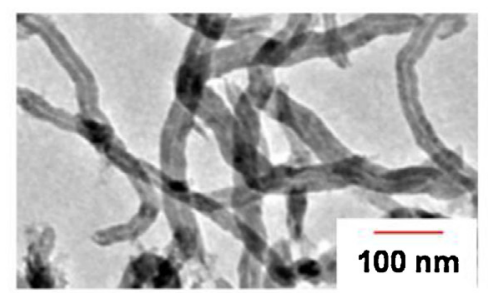

D

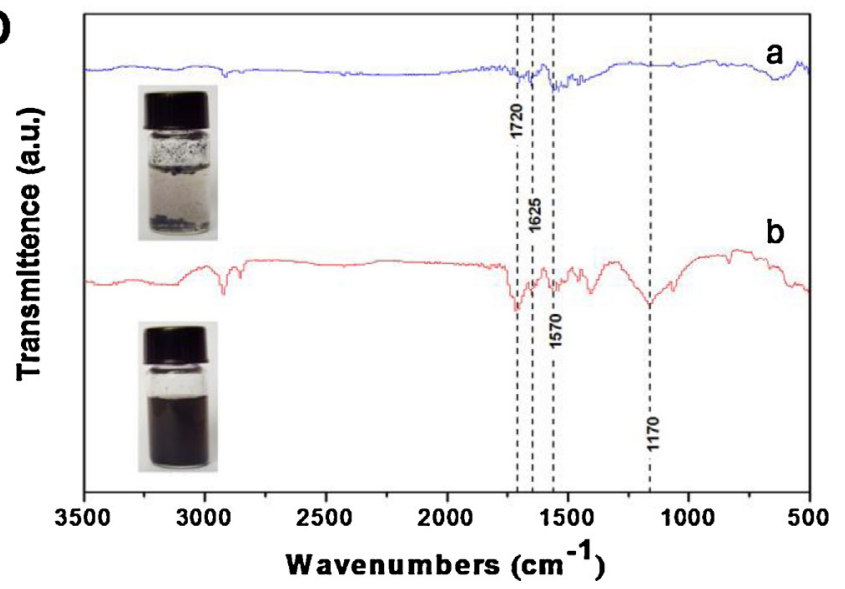

E

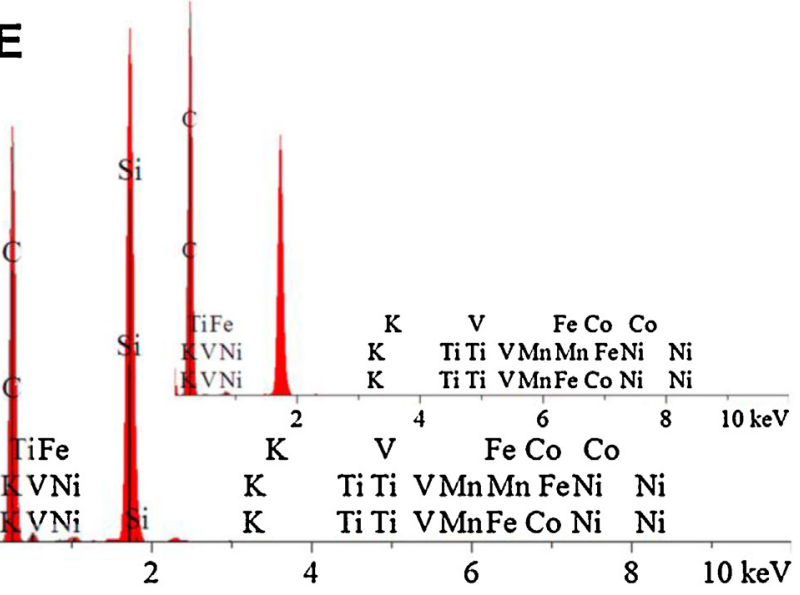

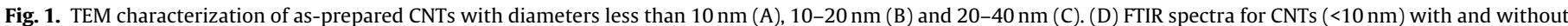
carboxylation. (E) EDX spectra of purified and raw (inset) nanotubes.
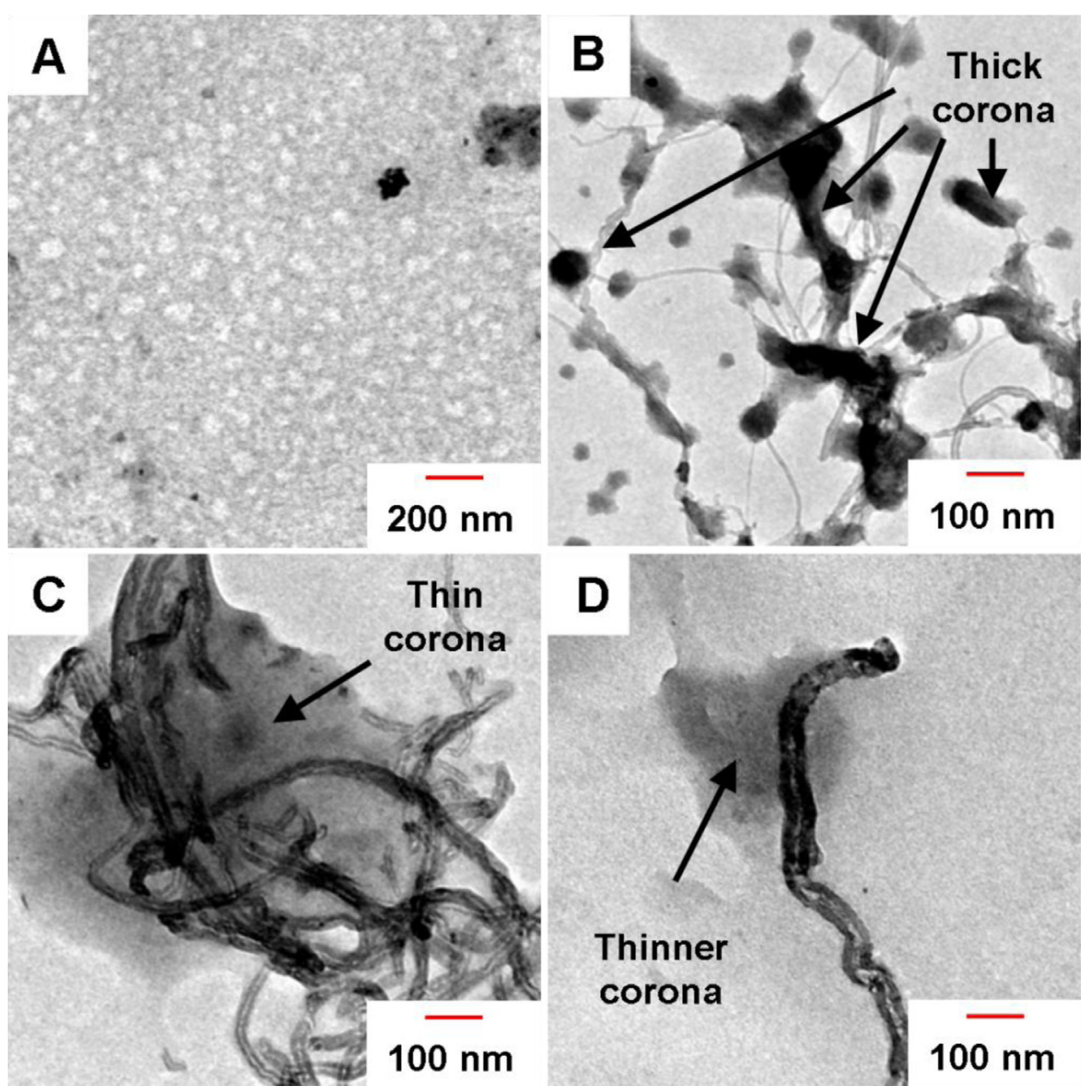

Fig. 2. TEM images of (A) BSA; the mixture of (B) $<10 \mathrm{~nm}$, (E) $10-20 \mathrm{~nm}$, and (F) $20-40 \mathrm{~nm}$ CNTs with BSA. 

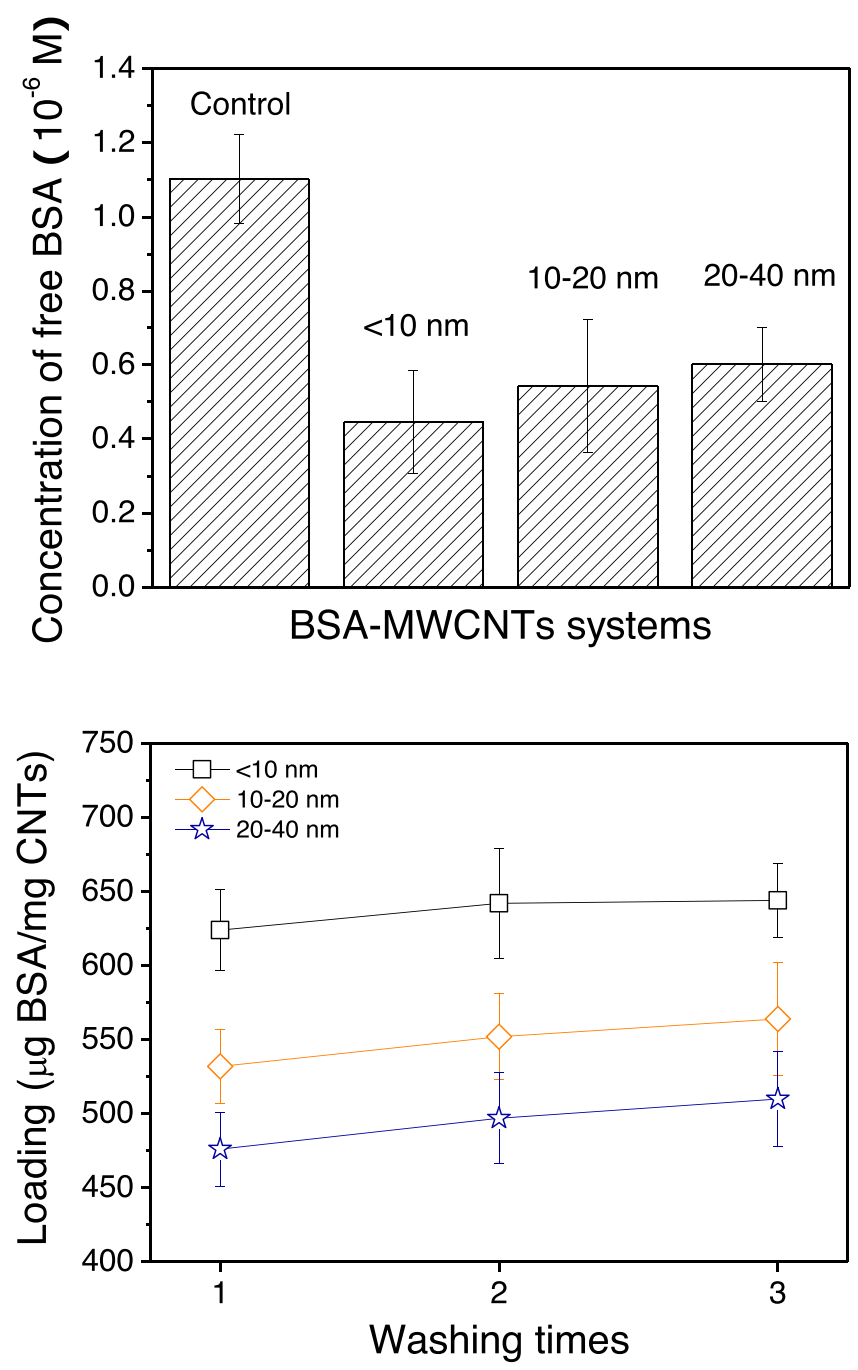

Fig. 3. Loading of BSA on CNTs and free protein concentration in the systems. Final concentrations of BSA and CNTs before filtration were $1.1 \times 10^{-6} \mathrm{M}^{-1}$ and $50 \mathrm{mg} / \mathrm{L}$, respectively. Each point was the average result of three separate experiment.

GIBCO, USA), and antibiotics (penicillin $100 \mathrm{U} / \mathrm{mL}$ and streptomycin $100 \mu \mathrm{g} / \mathrm{mL}, \mathrm{GIBCO}, \mathrm{USA})$ at $37^{\circ} \mathrm{C}\left(5 \% \mathrm{CO}_{2}, 95 \%\right.$ air $)$.

Briefly, astrocytes were seeded in 96-well microplates (Corning, USA) at a density of $1 \times 10^{4}$ cells per well. After cell growth by $80 \%$, plates were cultured in medium with $10 \mathrm{mg} / \mathrm{L}$ CNTs or CNT-BSA complex and $0.1 \%$ BSA for $24 \mathrm{~h}$. Astrocytes in culture media without CNTs was used as control. Cell viability was evaluated by the alarma blue staining assay. The fluorescence was measured at excitation wavelength of $530 \mathrm{~nm}$ and emission wavelength of $590 \mathrm{~nm}$ in a multiwell fluometric reader (Thermo, USA). Cell viability was calculated using equation: Cell viability $=($ Sample O.D. $) /($ Control O.D.) $\times 100 \%$

\section{Results and discussion}

\subsection{Characterization of as-prepared CNTs}

CNTs of different diameters were characterized by TEM and FTIR. Fig. 1A-C displayed the functionalized CNTs with diverse diameters ranges, which shows fine unity and homogeneity. The peak at $1570 \mathrm{~cm}^{-1}$ is associated with the $\mathrm{C}=\mathrm{C}$ stretching of CNT sidewall defects. The weak peak at $1625 \mathrm{~cm}^{-1}$ seen in the two spectra shown in this figure is assigned to traces of water in $\mathrm{KBr}$ used for making the pellet. The peak at $1720 \mathrm{~cm}^{-1}$ in Fig. $1 \mathrm{Db}$ and Fig. S1 (see
Supplementary data) clearly represent the $\mathrm{C}=\mathrm{O}$ stretching mode in the $\mathrm{H}_{2} \mathrm{SO}_{4} / \mathrm{HNO}_{3}$ treated CNTs and the strong absorption peak at about $1170 \mathrm{~cm}^{-1}$ is typical for the stretching of $\mathrm{C}-\mathrm{O}$, which all indicate successful modification of $\mathrm{COOH}$ groups on the uncapped ends of the graphene walls and to the edges of the holes [12]. As shown in Fig. S2 (see Supplementary data), Dynamic light scattering (DLS) measured hydrated radiuses are very large (98.7, 145.3 and $203.1 \mathrm{~nm}$ for three CNTs with increasing diameter; averaged from three runs) due to the rod-shaped structure of CNTs. However the results can be used to make comparison in size among the three types of CNTs [13]. TGA gave useful information about the functionalized CNTs, because most of the $\mathrm{COOH}$ moieties on the CNTs were thermally unstable. The pristine CNTs sample was stable and hardly decomposed below $550^{\circ} \mathrm{C}$, while the $\mathrm{CNTs}-\mathrm{COOH}$ experienced decomposition at $200-400^{\circ} \mathrm{C}$ (Supplementary data, Fig. S3). Previous study has shown that toxicity of CNTs may be attributed from metal catalyst impurities [14]. However, Energy Dispersive Spectrometer (EDS) data (Fig. 1E; Supplementary data, Table S1) here show very little metal content of CNTs before and after acid treatment. Besides, larger share of oxygen and sodium elements after modification of carboxyl attached to CNTs in the form of COONa.

\subsection{Characterization of CNTs and their complexes with BSA using TEM and DLS}

To ensure the purification of CNTs and that they were attached with protein, TEM observations of the CNTs before and after purification, with and without protein were carried out. Compared with raw CNTs, the carbon impurities were removed from the tubes and they became neater, tidier and dispersed after acidification and oxidation (Supplementary data, Fig. S4). As shown in Fig. 2B, BSA molecules aggregate together and are adsorbed firmly onto $<10 \mathrm{~nm}$ CNTs. Protein gets compacted to form hard and thick corona, making the electron beam hard to get through. Distinct configuration of the adsorbed protein was observed compared to the blurred ones in Fig. 2C and D, confirming the existence of stronger bindings and firmer adsorption of BSA onto the surface of CNTs with a smallerdiameter $[15,16]$. DLS measurements showed increased hydrated radius after adding the protein to CNTs $(98.7-125 \mathrm{~nm}$ for $<10 \mathrm{~nm}$ group, $145.3-171.7 \mathrm{~nm}$ for $10-20 \mathrm{~nm}$ group, and $203.1-219.8 \mathrm{~nm}$ for 20-40 nm group). The formation of protein-CNT bioconjugates may cause bundles formation or agglomeration of CNTs, and the attached protein also increase the width of CNTs.

\subsection{Protein adsorption onto CNTs}

The adsorption of proteins on nanoparticles is vital in nanomedicine, for example, even a single serum protein molecule on a gold nanosphere used in biomedical imaging may increase the size too much for renal clearance [17]. The amount of protein loaded onto the CNTs was determined by measuring the concentration of free protein before and after CNT exposure in buffer. Each group was washed with buffer for three times to calculate the total amount of loaded protein. Differences in free protein concentration and the loading amount are shown in Fig. 3. Adsorbed BSA had strong affinity for CNTs of smaller-diametered, with more loading amount and lower free protein concentration observed, which is in great agreement with the above findings.

\subsection{Fluorescence quenching of BSA by CNTs with different diameters}

Fluorescence of BSA is mostly attributed to three intrinsic fluorophores: Trp-Tyr and Phe. Of all the three intrinsic fluorophores, Trp moieties contribute more than $95 \%$ to the flu- 

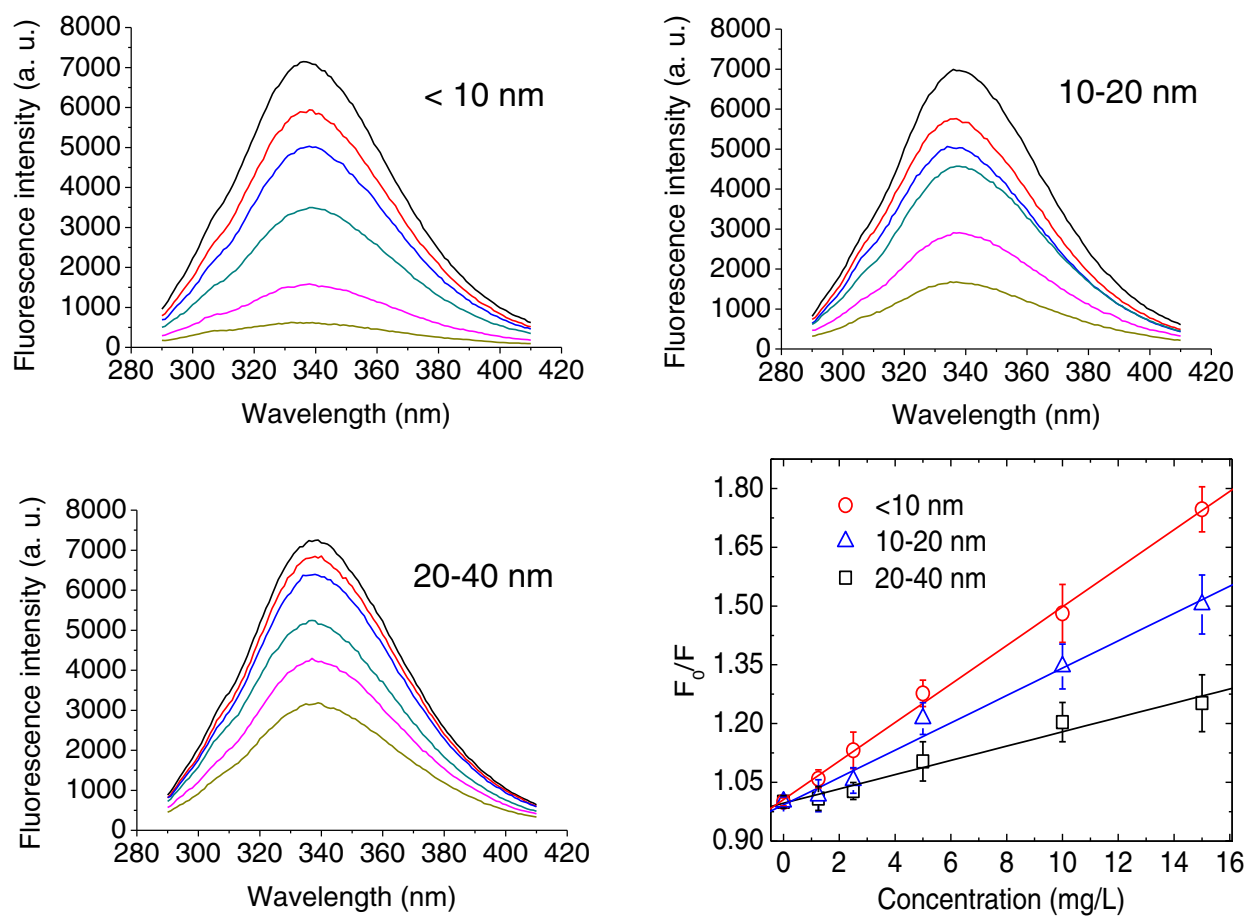

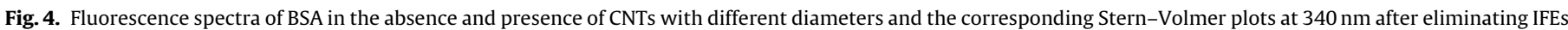
$C_{\mathrm{BSA}}=7.5 \times 10^{-7} \mathrm{M}^{-1}$, and $C_{\mathrm{CNTs}}$ (from top to bottom) $=0,2,4,6,8$ and $10 \mathrm{mg} / \mathrm{L}$, respectively.

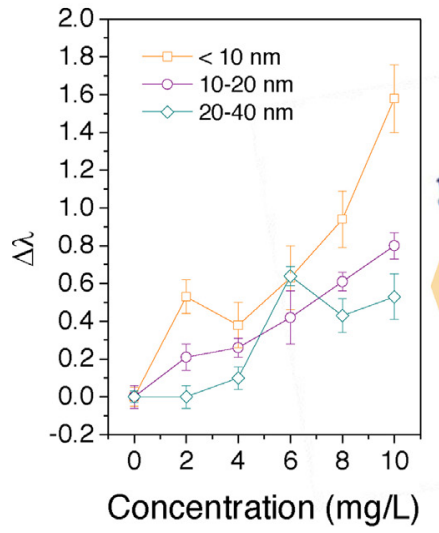

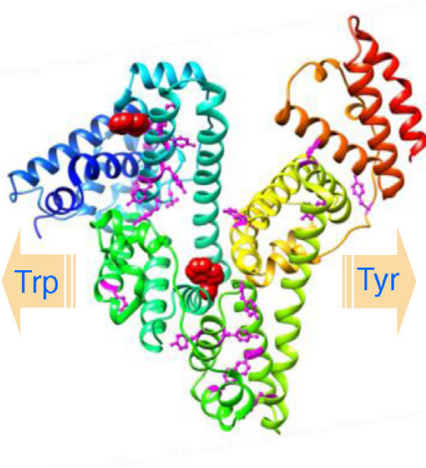

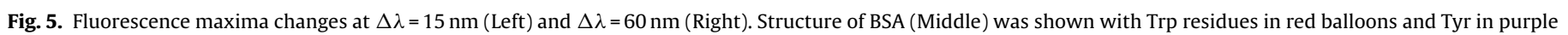
balls and bars.

orescence intensity. BSA possesses two tryptophan residues that have intrinsic fluorescence, Trp-134 in Domain I and Trp-212 in Domain II. Trp-212 is located within a hydrophobic binding pocket of the protein, and Trp-134 is on the surface of the molecule [18].

Fluorescence spectra are recorded in Fig. 4 in order to investigate the binding differences of CNTs to BSA due to diverse sizes. After the addition of different CNTs, the fluorescence spectra of BSA all experienced a quenching process, demonstrating alteration of BSA's structure and changes of intrinsic fluorophores' microenvironment [19]. With increasing diameter of CNTs, quenching of fluorescence becomes more evident and substantial. The increase of fluorescence quenching indicates that changes in the solventaccessible surface area of protein during its unfolding are greater for smaller-diameter nanotubes. These results demonstrate that the stability of BSA is strongly influenced by the diameter of CNTs.

\subsection{Synchronous fluorescence spectra}

Synchronous fluorescence spectroscopy has been widely used to investigate the conformational changes of protein molecules, for this spectroscopy effectively provides information on the vicinity of the fluorophore groups like Trp and Tyr residues. Fluorescence of proteins is known to come from chromophores molecules, and when the distinction between excitation and emission wavelength is set at $60 \mathrm{~nm}$, the fluorescence spectra are characteristics of Trp residues whereas $\Delta \lambda=15 \mathrm{~nm}$ indicates that of Tyr residues. The maximum emission wavelengths of Trp and Tyr residues in the protein molecule are related to the polarity of their surroundings, thus changes in maximum emission wavelengths are indicators of protein conformation. With the addition of CNTs the characteristic spectra of Trp and Tyr all experienced slight red shift after dislodging IFE. Therefore, the microenvironment of both Trp and 

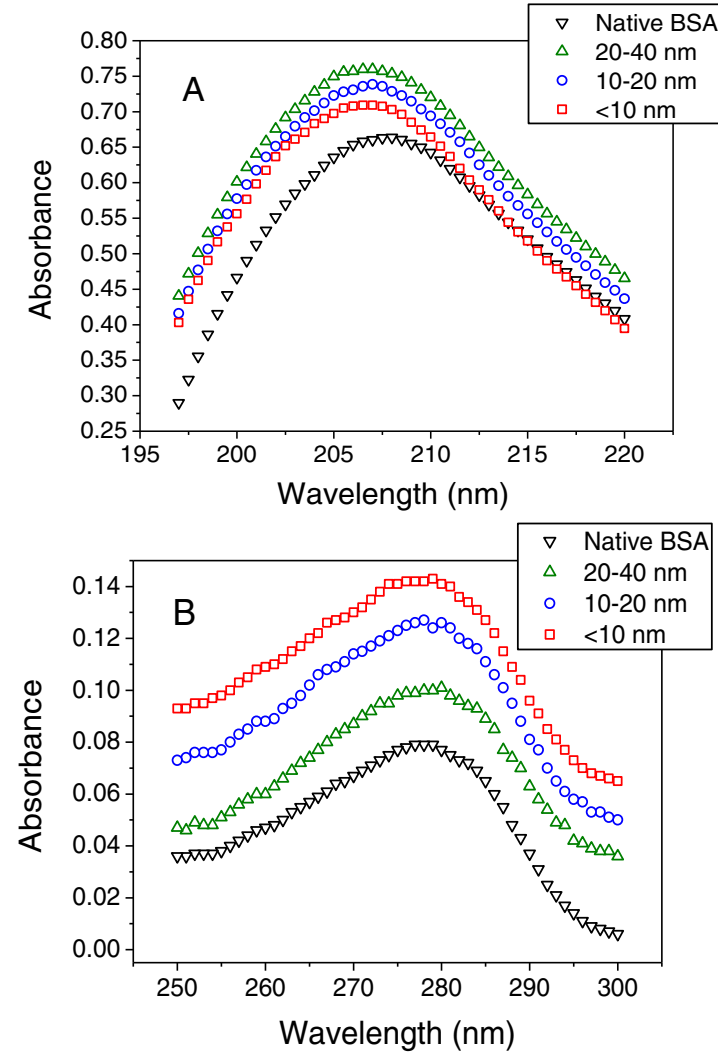

Fig. 6. UV-vis absorbance spectra of BSA with various CNTs. Final concentrations of BSA and CNTs were $5.6 \times 10^{-7} \mathrm{M}^{-1}$ and $2.5 \mathrm{mg} / \mathrm{L}$, respectively.

Tyr was disturbed by interaction of protein and the nanotubes. It is obvious that more bathochromic shifts occurred when $\Delta \lambda=60 \mathrm{~nm}$ is adopted with maxima ( $<10 \mathrm{~nm}$ group) shift of $1.6 \mathrm{~nm}$, while about $0.7 \mathrm{~nm}$ for $\Delta \lambda=15 \mathrm{~nm}$. This tendency was also observed in other groups (Fig. 5). Thus it can be safely concluded that the polarity around both Trp and Tyr has been changed and the effect of CNTs on the microenvironment around the Trp residues is stronger than that of Tyr. Besides, the intensity changes of Trp fluorescence is much stronger than that of Tyr (data not shown), implying the conformational changes of BSA are mainly attributed to the change of microenvironment around the Trp residues.

Unlike small molecules, before causing destruction to the Domains and Subdomains of BSA, CNTs tend to bring disturbance to its tertiary structure because of their steric hindrance (large size) and CNTs could not easily insert into the Domains before or without causing tertiary structural alteration. The BSA molecule possesses two Trp residues that possess intrinsic fluorescence: Trp-212 is located within a hydrophobic pocket of the protein, and Trp-134, on the surface of the albumin molecule. However, the Tyr residues, which are mostly deeply buried in the three domains of BSA, have few chances to be affected by the tubes. Thus, the Trp residues of BSA are easier to touch or be influenced by CNTs.

It is also observed that shifts at smaller-diametered groups are more apparent and we infer that CNTs with smaller diameter are more capable of accessing the fluorophores or more fluorophores (more BSA molecules) are attached to the surfaces of them.

\subsection{Diameter-dependent absorbance changes of the BSA-CNTS system}

UV-vis absorption measurement was used to explore the structural changes of BSA caused by CNTs. As can be seen in Fig. 6A, the strong absorption bands at around $208 \mathrm{~nm}$ were attributed to the transition of $\pi \rightarrow \pi^{*}$ of BSA's characteristic polypeptide backbone structure $\mathrm{C}=\mathrm{O}[20]$. It was recently found that $\mathrm{C}=\mathrm{O}$ bonds in protein could be recognized and be affected by the corresponding aromatic structure of CNTs [21]. The addition of CNTs induced absorbance rise of the protein, or rather distortion of the protein chain, which was caused by the hydrophobic surfaces of CNTs. One point to be noted is that the protein interacting with smaller CNTs has weaker absorbance. CNTs with smaller diameter create a smaller nonpolar environment for amide moieties of the adsorbed protein, leading to a higher energy gap of the transition, which caused a hypsochromic shift and the hyperchromic effect seen in the spectra; however, the BSA molecules get denatured more and the main chain becomes uncurled more on smaller CNTs, resulting in a greater possibility for the slack structure to encounter $\mathrm{H}_{2} \mathrm{O}$. These results correlated well with the observed stability changes from fluorescence outcomes. The absorption band around $280 \mathrm{~nm}$ was attributed to $n \rightarrow \pi^{*}$ electron transition of the chromophores. When CNTs were added to protein solution, the absorbance was enhanced and increased with decreasing diameter of CNTs (Fig. 6B). One possible explanation for the progressive enhancement in intensity is that BSA molecules have more chances to get loosened when adsorbed onto CNTs with smaller diameter. More protein molecules get denatured or the protein molecules are easier to denature when they contacted to the surface of the tubes, leading to structural alteration and exposure of the aromatic amino acid residues.

\subsection{CD analysis for protein secondary structure changes}

CD has become a convenient and widely employed method for the elucidation of the absolute conformation of chiral organic compounds in the past decades [22]. Here we applied this technique to investigate the secondary structure changes of BSA caused by CNTs. There is an increasing destructive effect of CNTs targeted to the secondary structures of BSA as the diameter of nanotubes decreases from $40 \mathrm{~nm}$ to less than $10 \mathrm{~nm}$, and the content of $\alpha$-helix decreased from $58.9 \%$ to $54.6 \%$ (Fig. 7). The $\alpha$-helix-rich substances are likely to have the hydrogen bonding networks destroyed, losing their $\alpha$-helical content and obtaining $\beta$-sheet content on smallerdiametered groups. Furthermore, a decrease in the percentage of $\beta$-turns and an increase in the unordered structure were observed, which suggested that the binding of CNTs to BSA induced unfolding of the polypeptides of protein.

\subsection{Zeta potential measurements of the systems}

Zeta potentials of BSA, CNTs, and CNT-protein corona were recorded at $\mathrm{pH} 4.5-8.5$ as shown in Fig. 8. As demonstrated in Fig. 8, at $\mathrm{pH}$ 7.4 BSA molecules bear negative charge surface with $\zeta$ potential of about $-6.86 \mathrm{mV}$ while $-45.9,-34.8$, and $-27.0 \mathrm{mV}$ for $<10$, 10-20 and 20-40 nm CNTs, respectively. Based on the fact that carboxylized CNTs interact with proteins through mainly electrostatic forces [23,24], it is assumed that the hydrophobic cavity of BSA, which is positive-charged at $\mathrm{pH} 7.4$, is the main site to interact with the negative-charged CNTs. Trp-212, which originally buried in the hydrophobic cavity of BSA, has high possibility of coming into contact with CNTs, causing synchronous fluorescence spectra shifts at $\Delta \lambda=60 \mathrm{~nm}$.

According to the measurements, the CNT surfaces are always negatively charged over the whole investigated $\mathrm{pH}$ range while BSA shows an isoelectric point at $\mathrm{pH}$ 4.6. BSA bears very little negative charge on their surface in the PBS buffer at $\mathrm{pH}$ above its isoelectric point. The negative charge developed in CNTs is assumed to be due to carboxyl groups at their surfaces as shown in Fig. 8. The zetapotential of the CNTs decreases with increasing $\mathrm{pH}$, which might result from the protonation of negatively-charged groups on the CNT surfaces. As stated previously, CNTs with smaller diameter are 

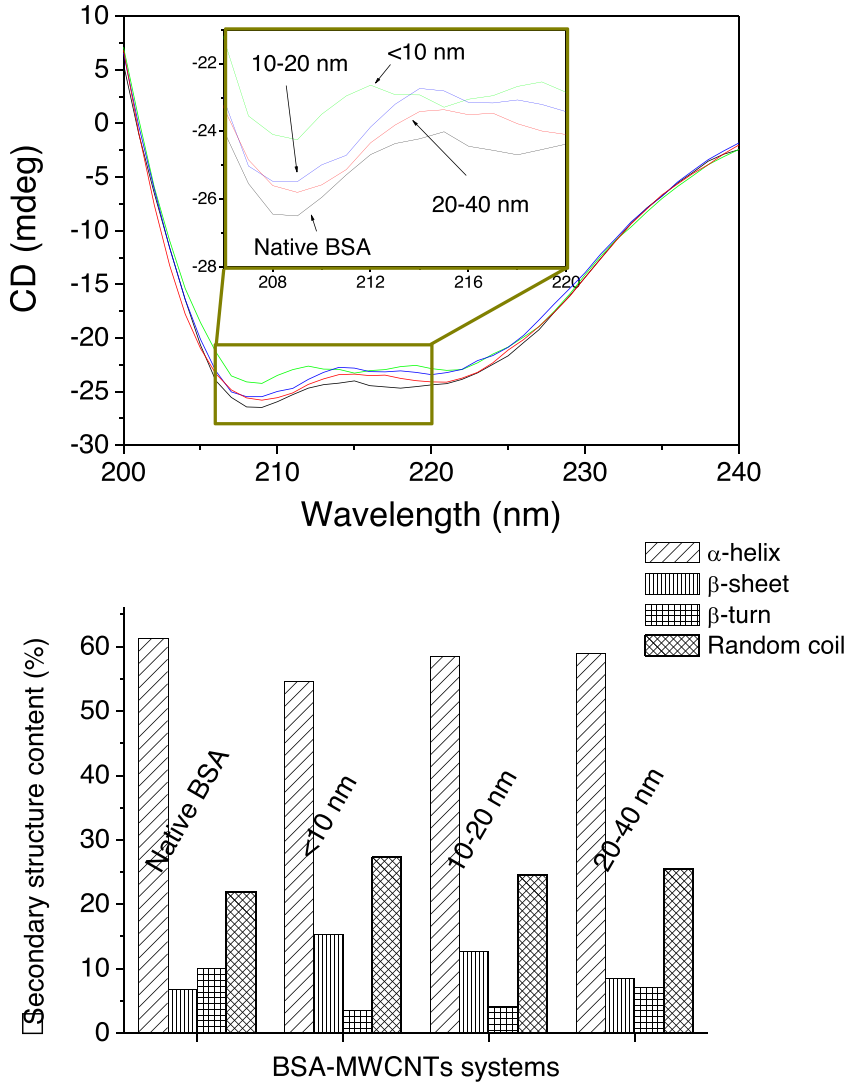

Fig. 7. CD spectra of BSA-CNTs system and content of $\alpha$-helix and $\beta$-sheet. Final concentrations of BSA and CNTs were $2 \times 10^{-7} \mathrm{M}^{-1}$ and $2 \mathrm{mg} / \mathrm{L}$, respectively.

known to have larger surface area, thus under similar modification conditions, a larger amount of carboxyl groups have been modified onto small-diametered CNT surfaces.

It is interesting to find that by adding BSA to CNT suspension the $\zeta$ potential is decreased to a level between those of the CNTs and BSA, and $\zeta$ potential of all the three groups reach the same level. The addition of BSA into CNT suspension gives rise to the formation of BSA-CNT complexes by virtue of the high affinity of CNTs with BSA. For the conformation of BSA-CNT complexes, it is possible that BSA is attached on the surface of CNTs, blocking the negative charges. In view of the fact that more protein is engaged in the binding event for small-diametered CNTs relative to large diameter ones, more surface charges on the former CNTs thus might be shielded by the adsorbed protein, leaving partial exposure of CNT surface. Besides, it is important to address that fraction of protein coverage for small-diametered CNTs is relatively higher because if the fraction of protein coverage is the same for the three groups, $<10 \mathrm{~nm}$ group should show more negative charge characteristics due to its larger share of original surface charge.

\section{9. $p H$-dependent conformational changes}

The tertiary structure of BSA consists of nine loops stabilized by 17 internal disulfide bonds between 34 cysteine residues, resulting in three primary domains each containing one small and two large loops. These disulfide bridges are the basis for the compact heart-shaped structure at physical pH [25]. From acidic to basic conditions, BSA is known to undergoe several transitions including the extended $(E)$, fast $(F)$, normal $(N)$, basic $(B)$ and aged $(A)$ forms. The $\mathrm{N}-\mathrm{F}$ isomerization involves unfolding and separation of domain III from rest of the molecule without significantly affecting rest of the molecule. Below $\mathrm{pH} 4$, an additional transition takes place resulting in a fully extended structure (the $\mathrm{E}$ form). At $\mathrm{pH}$ values above 8 , another transition occurs resulting in the $\mathrm{B}$ form. Unlike the above transitions, very subtle changes occur for the $\mathrm{N}-\mathrm{B}$ transition, where almost no secondary structure is lost [26].

The Trp of the native, monomeric protein at physical $\mathrm{pH}(7.4)$ exhibited maximum fluorescence intensity at $342 \mathrm{~nm}$. In the $\mathrm{F}-\mathrm{N}-\mathrm{B}$ $\mathrm{pH}$ region, BSA shows a two-step alteration in fluorescence intensity. At first, by increasing the pH from 3.5 to 6.5 , the fluorescence rises, which is aligned with a slight, but significant, increase in the emission maximum from 338 to $342 \mathrm{~nm}$ (Supplementary data, Fig. S5). By further increasing the $\mathrm{pH}$ to 8.5 , the fluorescence intensity decreases; however, no change in the position of the emission maximum is observed (Fig. 9A).

Under acidic conditions, the significant reduction of fluorescence is most likely to be attributed to the interaction of proton and Trp-212. As previous report clearly presented, fluorescence emission lifetimes of $\operatorname{Trp}$ residue differ at different $\mathrm{pH}$, indicating the presence of dynamic quenching of protein[27]. When $\mathrm{pH}$ transfers from 6.5 to 4.5 (pH region of the N-F transition, Fig. 9B), drastic structural change and rearrangement take place and the protein transforms to a molten-globule-like state[28], forming a less polar environment around Trp-212, which causes blue shifts in spectra. Under basic pH conditions, the tertiary and secondary structures suffer slight fluctuations. Both the fluorescence intensity and emission peak ( $343 \mathrm{~nm}$ ) reveal that Trp-212 is located close to the protein surface, and thus the crevice (between Domain I and III)

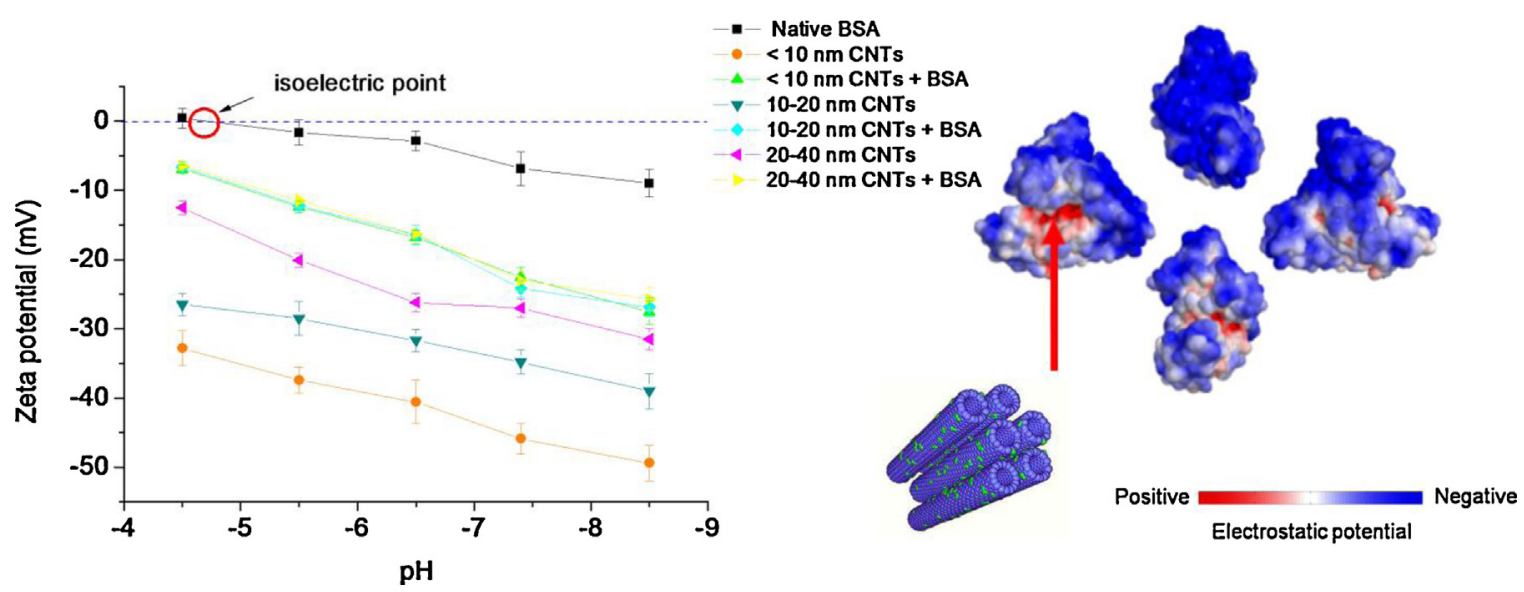

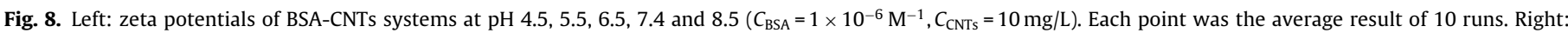
space-filling models of BSA and CNT models to indicate protein electrostatic surface potential at different degrees of rotation (pH 7.4) and the potential binding site. 


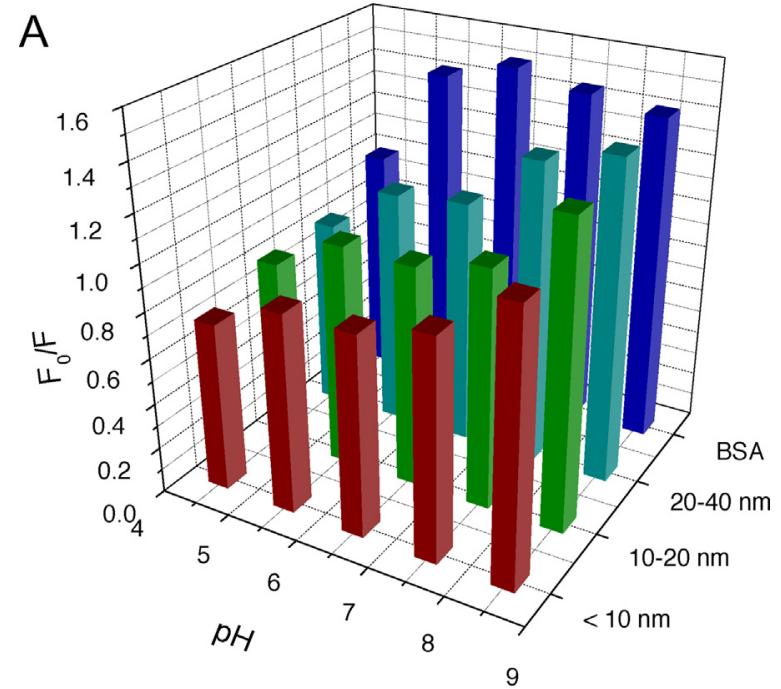

B

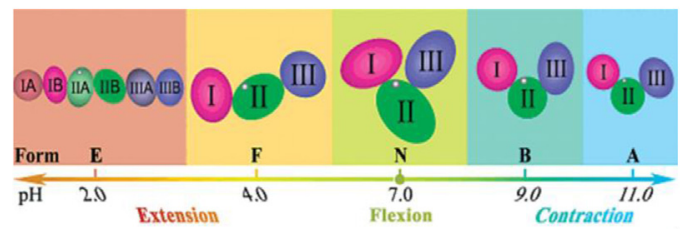

Fig. 9. (A) $F_{0} / F$ plots of $B S A-C N T$ systems at $340 \mathrm{~nm}$. Final concentrations of $B S A$ and CNTs were $1 \times 10^{-6} \mathrm{M}^{-1}$ and $10 \mathrm{mg} / \mathrm{L}$, respectively. (B) Schematic representation of conformational transitions, from the contracted configuration in basic $\mathrm{pH}$, to the flexible globule structure at neutral $\mathrm{pH}$, and to the extended form in acidic $\mathrm{pH}$. The location of Trp-212 is indicated by the white dot in domain IIA.

Source: adopted from Ref. [29].

must open widely to allow more mobile water molecules into the hydrophobic pocket[29].

Surprisingly, we observe significant increment of fluorescence density followed by slight blue shift when CNTs were introduced to BSA solution at $\mathrm{pH}$ range 6.5-8.5. The crevice of BSA is known to be hydrophobic and electropositive. With increasing $\mathrm{pH}$ the protein dynamically widens the crevice entrance and concurrently allows the globule structure to achieve more selective recognition, especially for our CNTs with hydrophilic and electronegative surfaces. After being bound to CNTs using the cavity structure, Trp-212 was placed to a more hydrophobic environmental matrix created by the protein and CNTs. Changes of the local environment around Trp212 therefore causes fluorescence enhancement along with blue shift.

\subsection{Cytotoxic effects of CNTs with and without BSA coating}

The potential toxicity of CNTs is a great issue which needs to be addressed for the safe use of this material. However, the role of proteins in particular on the toxicity effect of CNTs is only rarely investigated in contrast to their effects on biocompatibility. Carbon nanomaterials can cross the blood brain barrier (BBB) or transport from the olfactory nerve into the olfactory bulb [30]. Thus, they were speculated to accumulate and cause brain and nerve cell damage, or central nervous system (CNS) disorders [31]. Specially, CNTs have been shown to enter the CNS by sensory nerves or penetrating the BBB via olfactory nerve pathways and blood stream [32]. Astrocytes are the most numerous and diverse neuroglial cells in the central nervus system. They are responsible for creating the brain environment and building up the microarchitecture of the brain parenchyma. Therefore, it is very important to investigate the interaction between CNTs and the functionally important neuronal cells.

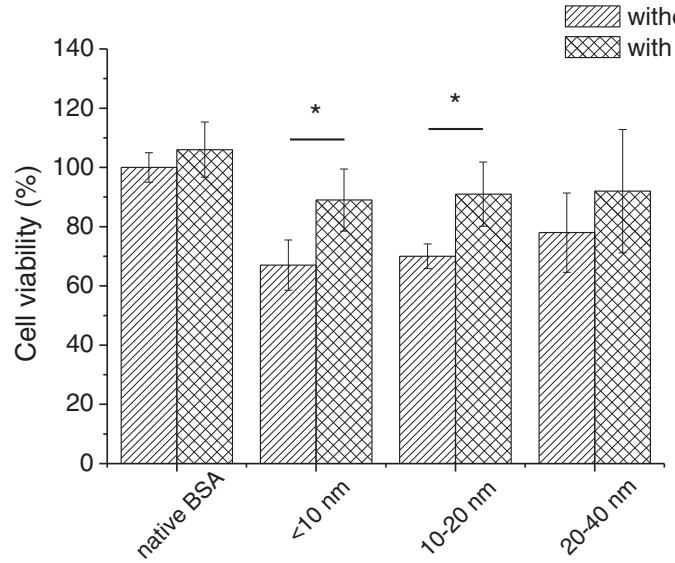

Fig. 10. Cytotoxicity of CNTs to cell line after $48 \mathrm{~h}$ incubation. Data are presented as mean \pm s.d. $(n=6)$. Statistically significant difference between groups: ${ }^{*} P<0.05$.

With decreasing CNT diameter, the cell viability increases gradually, implying that the impact of CNT diameter on the cell viability is considerable. CNTs with small diameter have more available surfaces for cells, thus have more changes to generate reactive oxygen species (ROS), which is fatal to cells [33]. After the addition of BSA, the cytotoxicity effects of CNTs were significantly reduced and cell viability increased from 67 to $89 \%, 70$ to $91 \%$, and 78 to $92 \%$ for the $<10 \mathrm{~nm}, 10-20 \mathrm{~nm}$ and $20-30 \mathrm{~nm}$ groups (Fig. 10), respectively. It can reasonably be concluded that BSA binds to CNTs causing a reduction of their cytotoxicity by forming CNT-protein conjugates. This viewpoint is supported by the results of recent studies where the attachment of serum albumin layer to nanomaterials reduces the biological toxicity of them whereas albumin-coated particles are less toxic [34,35].

It is interesting that significant reduction of cytotoxicity for smaller-diametered groups was found. This phenomenon might be related to the way of BSA adsorb onto CNTs. Distinct from the largediameter group, BSA can be attached to small-diametered CNT surfaces with the more compact form and the more layers, which may effectively protect cells from the exposure of CNT surfaces.

Since CNTs causes toxicity effects in the immediate proximity to the cells, the binding of BSA molecules to CNTs and the following coverage of their surfaces are key factors for their toxicity reduction. CNTs with protein corona coated have less surfaces exposed to media, therefore, less ROS may be generated. Besides, with improved biocompatibility, less CNTs may be endocytosed by the cells after BSA incubation.

\section{Conclusions and outlook}

In summary, diameter is a key factor of CNTs in influencing the binding of BSA onto them. The unfolding of BSA on CNTs is strongly affected by the diameter, hence surface area and surface curvature. Protein is less stable on CNT surfaces than in free solution, and the stability is decreased further on smaller-diametered nanotubes with larger surface area and curvature.

However, with regard to surface curvature, our research does conclude much on the behavior of single protein molecules on CNTs which have different surface curvatures, although a lot of works show surface-curvature-dependent protein binding to nanoparticles, the researchers always failed to consider or ignored surface area effects (the surface areas of these nanoparticles are not uniformly controlled, so it is not scientifically strict to talk about the surface-curvature-dependent effects). At a fixed mass concentration of nanoparticles, more protein was adsorbed on nanoparticles with smaller diameter. The results of the study are combinational 
effects of both surface area and surface curvature; however, the effect of surface area here evidently overwhelms that of surface curvature in terms of protein conformation.

As a potential influence element, surface area may play a key role in governing the interaction between protein and CNTs with diverse diameters. We intended to firstly prepare a series of stock CNT solutions with unique surface area, which are distinguished by diameters; and then repeat the measurements. Specific surface areas of the CNTs were determined by $\mathrm{N}_{2}$ adsorption using BET method. CNTs with smaller diameter were known to have larger specific surface areas; however, BET results (data not shown) went against with it. This situation is likely to be associated with the fact that long time of adsorption enables $\mathrm{N}_{2}$ molecules to penetrate into the graphene layers of MWCNTs, while BSA molecules, in contrast, cannot interact with MWCNTs on inner layers. Therefore, controlling the surface area of nanomaterials would make surface curvature the only variable in experiment, which can help reveal the effects of surface curvature.

Last but not least, since the reactivity of a protein is related to its conformation and the secondary structure, any structural change could seriously alter the activity or function of the protein. Structures of proteins are closely related to their activity and function. Disturbance on protein frame conformation may affect its physiological function, finally posing deleterious effects to organisms. Small-sized nanoparticles possess larger specific surface areas, which enable them to adsorb more amounts of proteins and provide them more contact area for biological membranes. On the other hand, the corona would reduce the direct contact of the nanoparticles with the cell membranes and protects the cells from the damages in the membrane caused by the toxic surface of the particles. Corona-coated nanoparticles may effectively prevent their surface from touching other proteins in ambient environment, and avoid dysfunction of these functional biomacromolecules in vivo or in vitro. Coating of CNTs with BSA significantly reduced their cytoxocity in vitro. Since CNTs show promising application in nanomedicine, preforming protein coated CNTs is expected to weaken opsonization and the reduced phagocytosis, and could be a versatile strategy for optimizing the CNT-based drug delivery systems.

\section{Acknowledgements}

We thank Dr. Xicheng Ma (School of Chemistry and Chemical Engineering, Shandong University) for the technical assistance on TEM. This work is co-supported by (20875055, 21277081, 21477067), the Cultivation Fund of the Key Scientific and Technical Innovation Project, Research Fund for the Doctoral Program of Higher Education, Ministry of Education of China (708058, 20130131110016), Science and Technology Development Plan of Shandong Province, China (2014GSF117027) are also acknowledged.

\section{Appendix A. Supplementary data}

Supplementary data associated with this article can be found, in the online version, at http://dx.doi.org/10.1016/j.jhazmat. 2015.03.023.

\section{References}

[1] S. Iijima, Helical microtubules of graphitic carbon, Nature 354 (1991) 56-58.

[2] A.H. Brozena, J. Moskowitz, B.Y. Shao, S.L. Deng, H.W. Liao, K.J. Gaskell, Y.H. Wang, Outer wall selectively oxidized, water-soluble double-walled carbon nanotubes, J. Am. Chem. Soc. 132 (2010) 3932-3938.

[3] Q.X. Mu, D.L. Broughton, B. Yan, Endosomal leakage and nuclear translocation of multiwalled carbon nanotubes: developing a model for cell uptake, Nano Lett. 9 (2009) 4370-4375.
[4] P. Bertoncini, O. Chauvet, Conformational structural changes of bacteriorhodopsin adsorbed onto single-walled carbon nanotubes, J. Phys. Chem. B 114 (2010) 4345-4350.

[5] D. Nepal, K.E. Geckeler, Proteins and carbon nanotubes: close encounter in water, Small 3 (2007) 1259-1265.

[6] R.F. Service, American chemical society meeting: nanomaterials show signs of toxicity, Science 300 (2003) 243.

[7] G. Brumfiel, Nanotechnology: a little knowledge, Nature 424 (2003) 246-248.

[8] B. Zhang, Y.H. Xing, Z.W. Li, H.Y. Zhou, Q.X. Mu, B. Yan, Functionalized carbon nanotubes specifically bind to alpha-chymotrypsin's catalytic site and regulate its enzymatic function, Nano Lett. 9 (2009) 2280-2284.

[9] Q.X. Mu, W. Liu, Y.H. Xing, H.Y. Zhou, Z.W. Li, Y. Zhang, L.H. Ji, F. Wang, Z.K. Si B. Zhang, B. Yan, Protein binding by functionalized multiwalled carbon nanotubes is governed by the surface chemistry of both parties and the nanotube diameter, J. Phys. Chem. C 112 (2008) 3300-3307.

[10] X.C. Zhao, R.T. Liu, Z.X. Chi, Y. Teng, P.F. Qin, New insights into the behavior of bovine serum albumin adsorbed onto carbon nanotubes: comprehensive spectroscopic studies, J. Phys. Chem. B 114 (2010) 5625-5631.

[11] M. Frangakis, H. Kimelberg, Dissociation of neonatal rat brain by dispase for preparation of primary astrocyte cultures, Neurochem. Res. 9 (1984) 1689-1698.

[12] J. Zhong, L. Song, J. Meng, B. Gao, W.S. Chu, H.Y. Xu, Y. Luo, J.H. Guo, A. Marcelli, S.S. Xie, Z.Y. Wu, Bio-nano interaction of proteins adsorbed on single-walled carbon nanotubes, Carbon 47 (2009) 967-973.

[13] T. Zhang, M. Xu, L. He, K. Xi, M. Gu, Z. Jiang, Synthesis, characterization and cytotoxicity of phosphoryl choline-grafted water-soluble carbon nanotubes, Carbon 46 (2008) 1782-1791.

[14] X. Liu, L. Guo, D. Morris, A.B. Kane, R.H. Hurt, Targeted removal of bioavailable metal as a detoxification strategy for carbon nanotubes, Carbon 46 (2008) 489-500.

[15] A. Cifuentes-Rius, H. de Puig, J.C.Y. Kah, S. Borros, K. Hamad-Schifferli, Optimizing the properties of the protein corona surrounding nanoparticles for tuning payload release, ACS Nano 7 (2013) 10066-10074.

[16] A. Rodriguez-Galvan, F.F. Contreras-Torres, E.V. Basiuk, E. Alvarez-Zauco, A. Heredia, V.A. Basiuk, Aggregation of human serum albumin on graphite and single-walled carbon nanotubes as studied by scanning probe microscopies, J. Nanosci. Nanotechnol. 11 (2011) 5491-5498.

[17] A.K. Murthy, R.J. Stover, W.G. Hardin, R. Schramm, G.D. Nie, S. Gourisankar, T.M. Truskett, K.V. Sokolov, K.P. Johnston, Charged gold nanoparticles with essentially zero serum protein adsorption in undiluted fetal bovine serum, J. Am. Chem. Soc. 135 (2013) 7799-7802

[18] P. Bourassa, C.D. Kanakis, P. Tarantilis, M.G. Pollissiou, H.A. Tajmir-Riahi, Resveratrol, genistein, and curcumin bind bovine serum albumin, J. Phys. Chem. B 114 (2010) 3348-3354.

[19] L.Z. Zhao, R.T. Liu, X.C. Zhao, B.J. Yang, C.Z. Gao, X.P. Hao, Y.Z. Wu, New strategy for the evaluation of CdTe quantum dot toxicity targeted to bovine serum albumin, Sci. Total Environ. 407 (2009) 5019-5023.

[20] R.T. Liu, F. Sun, L.J. Zhang, W.S. Zong, X.C. Zhao, L. Wang, R.L. Wu, X.P. Hao Evaluation on the toxicity of nanoAg to bovine serum albumin, Sci. Total Environ. 407 (2009) 4184-4188.

[21] J. Zhong, L. Song, J. Meng, B. Gao, W. Chu, H. Xu, Y. Luo, J. Guo, A. Marcelli, S. $\mathrm{Xie}, \mathrm{Z}$. Wu, Bio-nano interaction of proteins adsorbed on single-walled carbon nanotubes, Carbon 47 (2009) 967-973.

[22] J.N. Tian, J.Q. Liu, W.Y. He, Z.D. Hu, X.J. Yao, X.G. Chen, Probing the binding of scutellarin to human serum albumin by circular dichroism, fluorescence spectroscopy, FTIR, and molecular modeling method, Biomacromolecules 5 (2004) 1956-1961.

[23] N.W.S. Kam, H. Dai, Carbon nanotubes as intracellular protein transporters: generality and biological functionality, J. Am. Chem. Soc. 127 (2005) 6021-6026.

[24] H.J. Burch, S.A. Contera, M.R. de Planque, N. Grobert, J.F. Ryan, Doping of carbon nanotubes with nitrogen improves protein coverage whilst retaining correct conformation, Nanotechnology 19 (2008) 384001

[25] D.-H. Tsai, F.W. DelRio, A.M. Keene, K.M. Tyner, R.I. MacCuspie, T.J. Cho, M.R Zachariah, V.A. Hackley, Adsorption and conformation of serum albumin protein on gold nanoparticles investigated using dimensional measurements and in situ spectroscopic methods, Langmuir 27 (2011) 2464-2477.

[26] M. Bhattacharya, N. Jain, K. Bhasne, V. Kumari, S. Mukhopadhyay, pH-induced conformational isomerization of bovine serum albumin studied by extrinsic and intrinsic protein fluorescence, J. Fluoresc. 21 (2011) 1083-1090.

[27] M. Amiri, K. Jankeje, J.R. Albani, Characterization of human serum albumin forms with pH. Fluorescence lifetime studies, J. Pharm. Biomed. Anal. 51 (2010) 1097-1102.

[28] M. Dockal, D.C. Carter, F. Rüker, Conformational transitions of the three recombinant domains of human serum albumin depending on $\mathrm{pH}, \mathrm{J}$. Biol. Chem. 275 (2000) 3042-3050.

[29] W. Qiu, L. Zhang, O. Okobiah, Y. Yang, L. Wang, D. Zhong, A.H. Zewail, Ultrafast solvation dynamics of human serum albumin: correlations with conformational transitions and site-selected recognition, J. Phys. Chem. B 110 (2006) 10540-10549.

[30] A. Peters, B. Veronesi, L. Calderon-Garciduenas, P. Gehr, L.C. Chen, M. Geiser, W. Reed, B. Rothen-Rutishauser, S. Schurch, H. Schulz, Translocation and potential neurological effects of fine and ultrafine particles a critical update, Part. Fibre Toxicol. 3 (2006) 13.

[31] L. Meng, A. Jiang, R. Chen, C. -z. Li, L. Wang, Y. Qu, P. Wang, Y. Zhao, C. Chen, Inhibitory effects of multiwall carbon nanotubes with high iron impurity on 
viability and neuronal differentiation in cultured PC12 cells, Toxicology 313 (2013) 49-58.

[32] G. Oberdoerster, A. Elder, A. Rinderknecht, Nanoparticles and the brain: cause for concern? J. Nanosci. Nanotechnol. 9 (2009) 4996-5007.

[33] X. Zhao, R. Liu, Recent progress and perspectives on the toxicity of carbon nanotubes at organism, organ, cell, and biomacromolecule levels, Environ. Int. 40 (2012) 244-255.
[34] J. Bai, T. Wang Y. Wang X. Jiang, Effects of nanoparticle surface ligands on protein adsorption and subsequent cytotoxicity, Biomater. Sci. 2 (2014) 493-501.

[35] C. Ge, J. Du, L. Zhao, L. Wang, Y. Liu, D. Li, Y. Yang, R. Zhou, Y. Zhao, Z. Chai, Binding of blood proteins to carbon nanotubes reduces cytotoxicity, Proc. Natl. Acad. Sci. 108 (2011) 16968-16973. 